\title{
83. POLARIZATION OF SOUTHERN OB-STARS
}

\author{
G. KLARE and T. NECKEL
}

Landessternwarte and Max-Planck-Institut für Astronomie, Heidelberg-Königstuhl, Germany

The polarization data of 1421 southern OB-stars of the Heidelberg catalogue have been measured and plotted in a galactic $l^{\mathrm{II}}, b^{\mathrm{II}}$-diagram. For some longitude intervals the relationship of the standard deviation of the electric vector alignment and the galactic longitude was computed.

In 1966 a survey of OB-stars in the southern Milky Way has been completed at Heidelberg (Klare and Szeidl, 1966). 1660 objects between $l^{\mathrm{II}}=230^{\circ}$ and $l^{\mathrm{II}}=20^{\circ}$ could be identified with the use of 90 objective prism plates of the small Hamburger SchmidtSpiegel at Boyden. In earlier publications we have shown, that these OB-stars depict the spiral structure of the Milky Way up to distances of $4 \mathrm{kpc}$ from the sun, as shown in Figure 1 (Klare and Neckel, 1967). Also 21 OB-groups were found (Klare, 1967).

In January 1968 we began polarization- and UBV-magnitude measurements of all the OB-stars of the Heidelberg catalogue. We are using a 50-cm cassegrain reflector, which was built in Heidelberg and has been operating since autumn 1967 at the Boyden Observatory, South Africa. For polarization measurements a rotating polarization foil in the light path was used. Polarized light modulates the photo-current from the

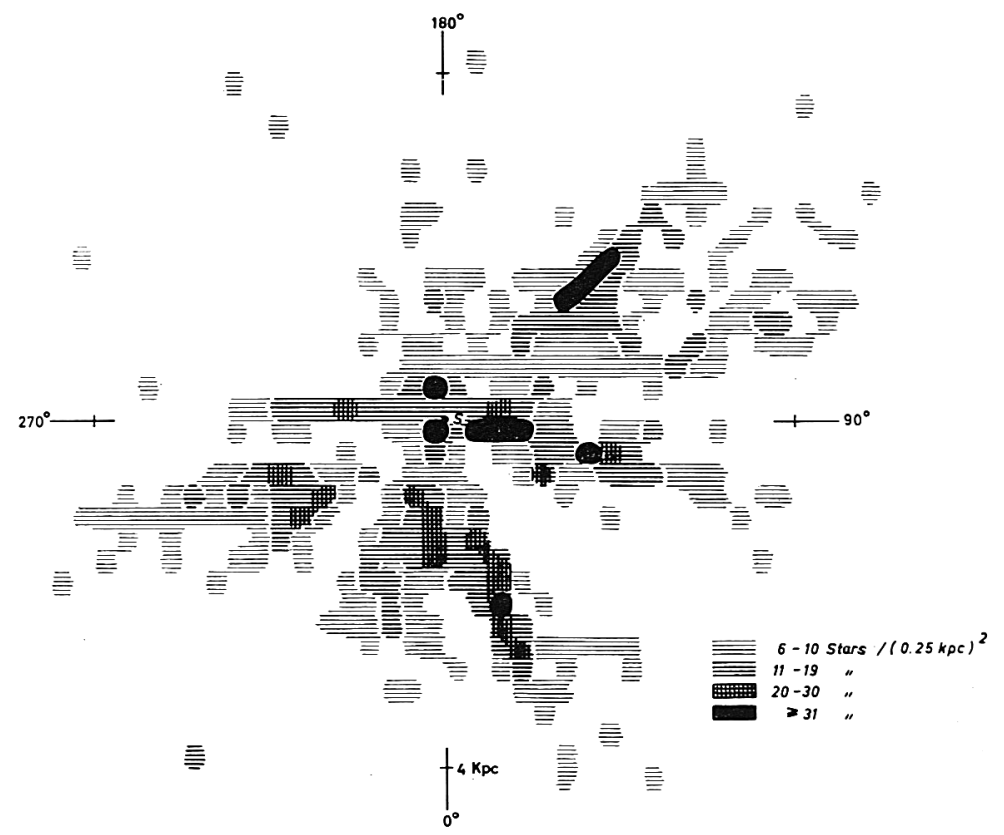

Fig. 1. Distribution of $5083 \mathrm{OB}^{+}$- and $\mathrm{OB}^{0}$-stars, and $1090 \mathrm{OB}$-stars with known MK-spectral type in the galactic plane. 
multiplier. The photo-current then goes through a voltage-to-frequency converter whose output impulses are counted by 3 counters. Two of the counters work only half a period and they are separated by a quarter of a period (Leinert et al., 1967).

To date we have measured the polarization of 1421 stars; a total of 2500 individual measurements were made. The accuracy of our measurements generally is 1 to $2 \%$ in the amount of polarization and $5^{\circ}$ in the polarization angle. We would like to point out, that the few stars, which have been observed as well by Hiltner as by us agree very well with each other.

We hope to be able to complete the polarization program within a few months. I will now discuss only the preliminary results.
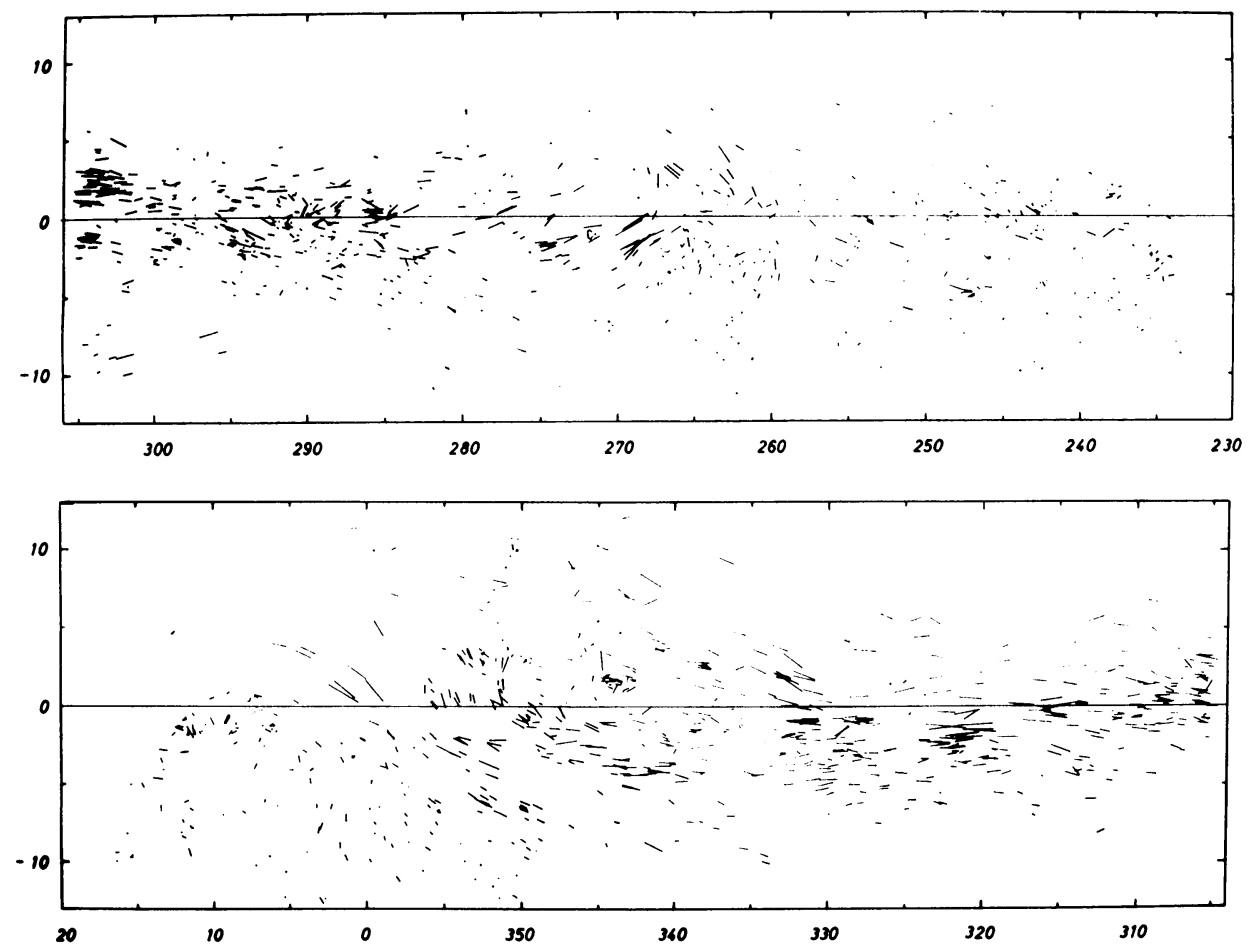

Fig. 2. The polarization of OB-stars plotted in a $l^{\mathrm{II}}, b^{\mathrm{II}}$-diagram. The length of the lines indicates the relative amount of polarization, the position angle indicates the plane of vibration.

Figure 2 contains all polarization values of the $1421 \mathrm{OB}$-stars. The longest lines represent degrees of polarization of 6 to $7 \%$.

Up to $l^{I I}=250^{\circ}$ the polarization is very small, which is not surprising, because in this direction we have very little absorbing matter. In the adjacent region to $l^{\mathrm{II}}=300^{\circ}$, the amount of polarization is larger, however, the directions of polarization show a large scatter. Between $l^{I I}=300^{\circ}$ and $l^{I I}=345^{\circ}$ the degree of polarization is very large and the direction very uniform and parallel to the galactic equator. From $l^{\mathrm{II}}=345^{\circ}$ 
to $l^{\mathrm{II}}=20^{\circ}$ we observe a whirling-like distribution, the directions of the polarization are partly vertical to the galactic equator, often they are inclined by $45^{\circ}$.

In Figure 3 we see the relationship of the standard deviation of the polarization angles from the mean value in some longitude intervals and the galactic longitude. Crosses represent the observations of Hiltner, and points represent our observations. The dot and the cross near $l^{11}=0^{\circ}$, which show a large discrepancy, cannot really be compared, due to a selection effect. The dot corresponds to many stars, distributed over a rather extended area, while the cross (Hiltner) corresponds only to a few stars concentrated on a small area, where the alignment of the electric vectors is quite uniform.

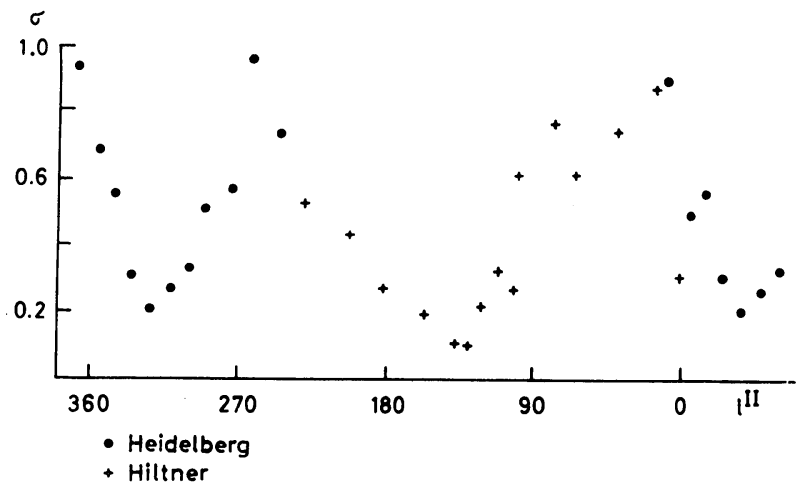

Fig. 3. The relationship of the standard deviation, $\sigma$, of the electric vector alignment and the galactic longitude.

According to the interpretation of Hiltner the maximum at $l^{\mathrm{II}}=80^{\circ}$ corresponds to the tangential direction of the local spiral arm in the solar neighbourhood; the minimum at $l^{\mathrm{II}}=140^{\circ}$ corresponds to that direction, in which the line of sight is vertical to the magnetic field.

In the southern Milky Way we observe a maximum in the standard deviation at $l^{I I}=260^{\circ}$. In this direction we expect to find - due to spiral arm indicators - the tangential direction of the local spiral arm, opposite to $l^{\mathrm{II}}=80^{\circ}$.

Also the minimum of the standard deviation in the southern sky at $l^{1 \mathrm{I}}=320^{\circ}$ lies opposite to the minimum in the northern sky, at $l^{\mathrm{II}}=135^{\circ}$.

\section{References}

Klare, G.: 1967, Z. Astrophys. 67, 249.

Klare, G. and Szeidl, B.: 1966, Veröff. Landessternwarte Heidelberg-Köningstuhl 18, 9.

Klare, G. and Neckel, T.: 1967, Z. Astrophys. 66, 45.

Leinert, C., Schmidt, T., and Schwarze, B.: 1967, Veröff. Landessternwarte Heidelberg-Königstuhl $20,23$. 